\title{
Inhibition of ovarian steroidogenesis by cyclic GMP in a fly
}

\author{
G Manière, E Vanhems, F Gautron and J-P Delbecque
}

Université Bordeaux I, Laboratoire de Neuroendocrinologie des Insectes, Avenue des Facultés, F-33405 Talence Cedex, France

(Requests for offprints should be addressed to J-P Delbecque who is now at Université Bordeaux I, Laboratoire de Neurobiologie des Réseaux, CNRS UMR 5816, Avenue des Facultés, F-33405 Talence Cedex, France; Email: jp.delbecque@Inr.u-bordeaux.fr)

(G Manière is now at CNRS, Institut de Neurobiologie Alfred Fessard, UPR 2216, Neurobiologie Génétique et Intégrative, Equipe de Génétique Moléculaire des Rythmes Circadiens, Avenue de la Terrasse, F-91198 Gif sur Yvette Cedex, France)

(F Gautron is now at Université Bordeaux I, Laboratoire des Facteurs de Croissance et de la Différenciation Cellulaire, INSERM EPI-0113, Avenue des Facultés, F-33405 Talence Cedex, France)

\begin{abstract}
Previous investigations in the female blowfly Phormia regina have shown that 3-isobutyl-1-methylxanthine (IBMX), a broad spectrum inhibitor of phosphodiesterases (PDEs), fails to mimic the steroidogenic effects of cAMP on ovaries, although it efficiently increases the concentrations of this second messenger. In this study, experiments carried out to clear up this contradiction demonstrated that IBMX, besides its effect on cAMP, also increased cGMP concentrations in blowfly ovary and that these two cyclic nucleotides controlled ovarian steroidogenesis antagonistically. In particular, a selective inhibitor of cGMP-specific PDEs, unlike IBMX, had a very strong negative effect on ovarian steroidogenesis. Moreover, a cGMP analog was able to inhibit steroid biosynthesis in previtellogenic and vitellogenic ovaries, thus affecting basal and acute steroidogenesis respectively. Our observations also demonstrated that cGMP was always present in blowfly ovary, reaching its maximal levels at the end of
\end{abstract}

vitellogenesis, in close correlation with the physiological decrease in ovarian steroidogenesis. Experiments using an inhibitor of protein kinase $G$ clearly indicated that the effects of cGMP were mediated by this enzyme. On the contrary, these effects did not seem to involve cGMPregulated PDEs or ion channels. Our results also indicated that ovarian cGMP concentrations were not controlled by brain factors, suggesting a probable involvement of paracrine/autocrine factors. Nitric oxide (NO) appeared to be a good candidate for such a control, because an $\mathrm{NO}$ donor was able to stimulate ovarian cGMP concentrations and to drastically decrease ovarian ecdysteroid biosynthesis in blowflies. These data thus demonstrate, for the first time in invertebrates, a potent role of cGMP in the negative control of ovarian steroidogenesis and suggest a possible co-regulation with NO.

Journal of Endocrinology (2003) 177, 35-44

\section{Introduction}

Steroidogenesis is often activated by peptidic hormones, which bind to specific membrane receptors on endocrine gland cells and exert their stimulatory action via the involvement of second messengers, in particular cAMP and calcium (for review see Cooke 1999). However, other signaling molecules, either stimulatory or inhibitory, also intervene for a fine tuning of this vital biosynthesis. Among them, cGMP, an ubiquitous second messenger involved in numerous physiological responses (for review see Drewett \& Garbers 1994, Lucas et al. 2000) may also have a modulatory role on steroidogenesis. Furthermore, cGMP is frequently linked with another important messenger, nitric oxide (NO), which is known to be a potent stimulator of soluble guanylate cyclases (for review see Bredt \& Snyder 1994) and thus a possible regulator of steroid biosynthesis.
Regarding more particularly the control of steroidogenesis in the gonads, the effects of cGMP have been studied in several vertebrate models, but not yet in invertebrates. In the former, however, the situation appears very confused: indeed, stimulatory effects of cGMP analogs have been sometimes observed (Mukhopadhyay et al. 1986, Khurana \& Pandey 1993) and interpreted as resulting from an interference with the protein kinase A pathway (Schumacher et al. 1992); conversely, inhibitory effects of cGMP have also been described (LaPolt \& Hong 1995) and related to a potential stimulation of soluble guanylate cyclases by $\mathrm{NO}$ (Welch et al. 1995, Grasselli et al. 2001, Ishimaru et al. 2001). However, in numerous other cases, it has also been observed that cGMP has no significant effect on steroidogenesis in gonads and that $\mathrm{NO}$ is able to play an inhibiting role alone, directly at the level of P-450 enzymes (Van Voorhis et al. 1994, Del Punta et al. 1996, Masuda 
et al. 1997). In this complex context, the study of invertebrate models may be of great interest for a comparative point of view.

In arthropods, steroid hormones, known as ecdysteroids or molting hormones, play a pivotal role not only in the control of embryonic and postembryonic development, but also in the regulation of their reproductive physiology. In particular, ecdysteroids are known to stimulate vitellogenin biosynthesis in the fat body of higher Diptera (for review see Hagedorn 1985). Biosynthesis of these hormones occurs in specialized glands known as molting glands, but also in several other sources (for review see Delbecque et al. 1990) and particularly in gonads, as demonstrated in adult insects (Hagedorn et al. 1975, Loeb et al. 1982). In insect molting glands (also known as prothoracic glands), steroidogenesis is mainly regulated by stimulatory brain peptides acting through calcium and cAMP (for review see Gilbert et al. 1996), whereas in crustacean molting glands (also known as Y-organs), it is negatively regulated by molt-inhibiting hormones (MIHs), which seem to act through either cGMP or cAMP (Sedlmeier \& Fenrich 1993, Spaziani et al. 1999).

In our previous studies on the control of steroidogenesis and vitellogenesis in our model, the blowfly Phormia regina, we have demonstrated that ovarian biosynthesis of ecdysteroids is stimulated by several (unidentified) brain factors, triggering either cAMP-dependent or cAMPindependent signaling cascades (Manière et al. 2000) and is also subjected to an unusual inhibitory control by calcium (Manière et al. 2002). During these investigations, we also observed that 3-isobutyl-1-methylxanthine (IBMX), a broad spectrum inhibitor of phosphodiesterases (PDEs), failed to mimic the steroidogenic effects of cAMP analogs or of forskolin, whereas some specific PDE inhibitors were able to do so (Manière et al. 2002). Attempts to elucidate this paradox have led us to the hypothesis that IBMX could have simultaneously two opposite pharmacological effects on steroid biosynthesis in blowfly ovaries, a stimulatory effect via the cAMP-signaling pathway and an inhibitory effect, possibly via cGMP involvement. The following study was thus undertaken to test this hypothesis and to investigate the potential role of cGMP in the control of ovarian steroidogenesis in blowflies.

\section{Materials and Methods}

\section{Animals}

Phormia maggots were regularly purchased from La Verminière de l'Ouest (Tremblay, France) and reared to adulthood as previously described (Manière et al. 2000). Adult blowflies (both sexes together) were maintained in controlled conditions $\left(25^{\circ} \mathrm{C}, 16 \mathrm{~h}\right.$ light: $8 \mathrm{~h}$ darkness cycle) and supplied with only sugar and water, this diet being known to prevent females from undertaking vitellogenesis and their oocytes from growing. Our experiments were performed on such sugar-fed females (named hereafter previtellogenic females), 5 to 7 days after adult eclosion, or on females which, after a similar sugar diet for 5-7 days, received a single protein meal (bovine or porcine liver) for $1 \mathrm{~h}$ or more; such a diet was necessary and sufficient to induce steroidogenesis and vitellogenesis. Liver-fed females were then named vitellogenic females and, unless otherwise stated, they were generally used $24 \mathrm{~h}$ after the meal. For more details, see Manière et al. (2000). Before any operation, flies were first anesthetized with $\mathrm{CO}_{2}$ and then maintained on crushed ice.

\section{In vitro experiments}

Ovaries from the same pair were extirpated under sterile conditions, rinsed four times and incubated separately in $50 \mu \mathrm{l}$ culture medium, using multiwell plates. Incubations were made at $26 \pm 1{ }^{\circ} \mathrm{C}$ in Grace's insect culture medium (Gibco, Life Technologies-France, CergyPontoise, France). Some experiments were also carried out using a defined saline solution without calcium $(128 \mathrm{mM}$ $\mathrm{NaCl}, 5 \mathrm{mM} \mathrm{KCl}, 2 \mathrm{mM} \mathrm{MgCl}, 1 \mathrm{mM} \mathrm{Na} \mathrm{HPO}_{4}$, $0.34 \mathrm{mM} \mathrm{KH}_{2} \mathrm{PO}_{4}, 26.8 \mathrm{mM}$ glucose, see Manière et al. 2002). Our biotests were made as previously described (Manière et al. 2000): in general, one ovary from an individual pair was tested for its response to a precise pharmacological treatment, whereas the contralateral ovary was used as its corresponding untreated control. These ovaries were generally named, respectively, treated and control ovaries. In some cases, two ovaries from the same pair were incubated separately in pure medium and were designated as blanks. After an appropriate incubation time $(1 \mathrm{~h}$ for cyclic nucleotides, $3 \mathrm{~h}$ for ecdysteroids in order to take account of their slower anabolism), ovaries were removed for the individual determination of cAMP and cGMP concentrations, whereas ecdysteroids were measured in incubation media.

Preparation of crude brain extracts was made as previously described (Manière et al. 2000): adult 'brains' (defined here as the whole cephalic nervous mass, including subesophageal ganglion) were extirpated under sterile conditions, either from previtellogenic or vitellogenic females. Pools of approximately 50 brains were homogenized in Grace's insect culture medium (at a final concentration of three brain equivalents in $50 \mu$ medium, i.e. per culture) and were centrifuged; then the supernatants were filtered on $0.2 \mu \mathrm{m}$ sterile cartridges (Millipore, St Quentin en Yvelines, France).

\section{Chemicals}

All chemicals used in this study were purchased from Sigma (distributed by Sigma-Aldrich-France, St Quentin Fallavier, France), except $4-\left\{\left(3^{\prime}, 4^{\prime}-\right.\right.$ (methylenedioxy)benzyl)amino $\}$-6-methoxy-quinazoline (MDBAMQ) from Calbiochem (France Biochem, 
Meudon, France) and erythro-9-(2-hydroxy-3nonyl)adenine (EHNA) from RBI (distributed by SigmaAldrich-France). When necessary, chemicals were previously dissolved as concentrated stock solutions in dimethyl sulfoxide or $50 \%$ ethanol, then appropriately diluted into the culture medium: in these cases, a similar amount of solvent was added in the corresponding control incubations.

\section{Immunoassays}

Ecdysteroids, cAMP and cGMP were measured using immunoassays. Ecdysteroids were measured as previously described (Manière et al. 2000), from the incubation medium of a single ovary, by enzyme immunoassay using L2 antibody. This polyclonal antibody, raised against an ecdysone conjugate, is particularly convenient for ecdysone measurements (Pascual et al. 1995); it was chosen as being the most suitable for our experiments among various other antibodies with different specificities (authors' unpublished observations). Results were obtained by comparison with calibrated solutions of ecdysone and thus expressed as fmol ecdysone equivalents per culture. Taking account of sample dilution (which was made in order to measure them several times if necessary), the inferior limit of sensitivity of our assay was approximately $2 \mathrm{fmol} /$ ovary.

cAMP was also measured as previously described (Manière et al. 2000), after extraction of a single incubated ovary and acetylation of the extract, using the immunoassay of Kingan (1989). Results were obtained as fmol/ ovary. The inferior limit of sensitivity of this method was approximately 1-2 fmol/ovary.

cGMP was measured using RPN-226 enzyme immunoassay kits from Amersham-Pharmacia (Orsay, France). The extraction and acetylation of extracts were similar to those used with cAMP (Kingan 1989). The rest of the protocol followed the recommendations given with the commercial kits. Results were also obtained as fmol/ ovary. The inferior limit of sensitivity of this method was also 1-2 fmol/ovary.

\section{Presentation of results and statistics}

The majority of the results obtained after immunoassay measurements are presented as means ( \pm S.E.) calculated from the individual ratios obtained by dividing the concentration obtained from one ovary with that obtained from the corresponding contralateral one. Such concentration ratios (or treated/control ratios), independent of the size of flies (or of ovaries) and of incubation times, appeared more convenient than absolute amounts for comparisons between various experiments (see Manière et al. 2000, for more details). Statistical analysis was generally made using Student's $t$-tests by comparing means of concentration ratios to the theoretical value of 1 (equivalent to a paired test).

\section{Results}

\section{Effects of IBMX and of cGMP-mimicking compounds}

In order to confirm our hypothesis according to which IBMX could activate simultaneously both cyclic nucleotide-signaling cascades in our model, measurements of cAMP and cGMP, together with ecdysteroids, were performed from blowfly previtellogenic ovaries explanted in vitro, in the absence or the presence of IBMX. At first, blanks in the absence of IBMX verified an important property of this model: when explanted separately in vitro, previtellogenic ovaries from the same pair (Fig. 1A) maintained very similar concentrations of cGMP, as well as of cAMP, and secreted the same amounts of ecdysteroids in the medium (means of individual concentration ratios between ovaries of the same pair were not significantly different from 1, Student's $t$-test, $P>0 \cdot 05$ ). Then, when similar ovaries (one per pair) were individually treated with IBMX $\left(10^{-3} \mathrm{M}\right)$ and compared with their respective contralateral untreated ovaries (Fig. 1B), they presented a significant increase of both cyclic nucleotides (ratios significantly higher than 1 , Student's $t$-test, $P<0 \cdot 05$ ), whereas ecdysteroid secretion remained unchanged (ratio not significantly different from $1, P>0 \cdot 05$ ).

In order to investigate the potential role of cGMP, a cGMP analog, chloro-phenyl-thio-cGMP (CPT-cGMP), was tested on previtellogenic ovaries (explanted from sugar-fed females), as well as on vitellogenic ones (explanted from liver-fed females $24 \mathrm{~h}$ after the beginning of the protein meal). Results, given in Fig. 2A, show that this cGMP analog had a significant inhibitory effect on steroidogenesis (at $10^{-5}$ and $10^{-4} \mathrm{M}$ ) in both previtellogenic and vitellogenic ovaries. Blank experiments (results given at concentrations 0 ) also verified that both ovaries from similar flies, either in previtellogenesis or in vitellogenesis, secreted the same quantities of ecdysteroids (ratios not significantly different from $1, P>0 \cdot 05$ ). Then, experiments with a selective inhibitor of cGMP-specific (type V) phosphodiesterases, MDBAMQ (Takase et al. 1994), were performed on previtellogenic as well as on vitellogenic ovaries. Figure $2 \mathrm{~B}$ shows that this specific inhibitor, unlike IBMX, reduced ecdysteroid secretion (at $10^{-5}$ and $10^{-4} \mathrm{M}$ ) very efficiently in vitellogenic and, to a lesser extent, in previtellogenic ovaries.

CTP-cGMP and, more efficiently, MDBAMQ (Fig. 3) were also able to reduce the stimulation of ovarian ecdysteroid biosynthesis triggered by a cAMP-mimicking compound such as forskolin (used here at the optimal concentration of $10^{-5} \mathrm{M}$, see dose-response curve in Manière et al. 2000). Interestingly, a capacity to inhibit steroidogenesis was also revealed for IBMX in the 


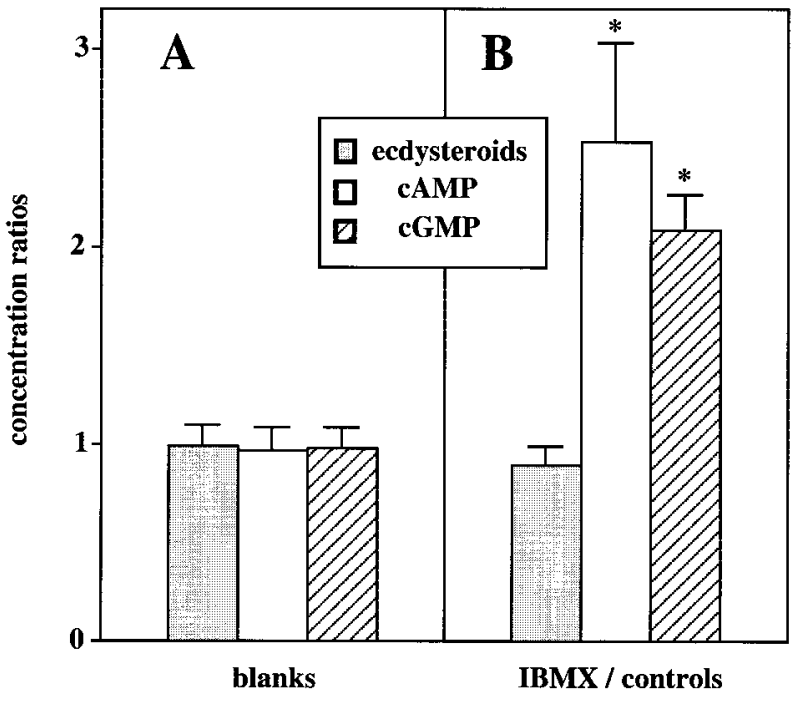

Figure 1 Effects of the absence (A) or the presence (B) of IBMX on ecdysteroid, CAMP and cGMP biosyntheses by previtellogenic ovaries in vitro. Ovaries were incubated in Grace's medium for $1 \mathrm{~h}$ for CAMP and cGMP measurements or $3 \mathrm{~h}$ for ecdysteroid measurements. Each bar represents a mean \pm S.E.M. obtained from six to eight pairs of ovaries. The set without IBMX (A) was made to verify whether ecdysteroids, CAMP and CGMP were synthesized in equivalent amounts in both ovaries of the same pair. For IBMX treatment (B), $10^{-3} \mathrm{M}$ IBMX was added to one ovary of each pair and the contralateral one was used as its corresponding untreated control. Results (ordinate) are expressed by dividing ecdysteroid quantities secreted by the two ovaries of the same pair for series $A$, in order to obtain treated/control ratios for series $\mathrm{B}$. The ordinate represents the means of these ratios and asterisks above error bars (standard errors) indicate results significantly different from the theoretical value of 1 (Student's $t$-test, $P<0 \cdot 05)$. When expressed in absolute amounts, ecdysteroids secreted into the medium after a 3 -h incubation of untreated ovaries (controls) represent $11 \cdot 3 \pm 2 \cdot 5 \mathrm{fmol}$ ecdysone equivalents per ovary $(n=8)$, whereas internal concentrations of cyclic nucleotides (after $1 \mathrm{~h}$ of incubation) represent $12 \cdot 8 \pm 3 \cdot 7 \mathrm{fmol}$ cAMP per ovary $(n=8)$ and $13 \cdot 5 \pm 1 \cdot 8 \mathrm{fmol}$ cGMP per ovary $(n=8)$.

presence of forskolin: IBMX (at $10^{-3} \mathrm{M}$ ) even appeared to be as efficient as $10^{-4} \mathrm{M}$ CTP-cGMP in reducing forskolin stimulation. Altogether, these observations suggested that an inhibitory effect of IBMX on ovarian steroidogenesis, obviously due to a rise in cGMP concentrations, can be observed in particular conditions such as an excess of cAMP, but that this effect is not visible when cAMP and cGMP concentrations rise with a similar intensity.

\section{Variations in cGMP concentrations during the vitellogenic cycle}

Time correlations between variations in ovarian concentrations of cGMP and changes in ecdysteroid biosynthesis were examined after a protein meal. In particular, comparisons were made between the cGMP content of indi-

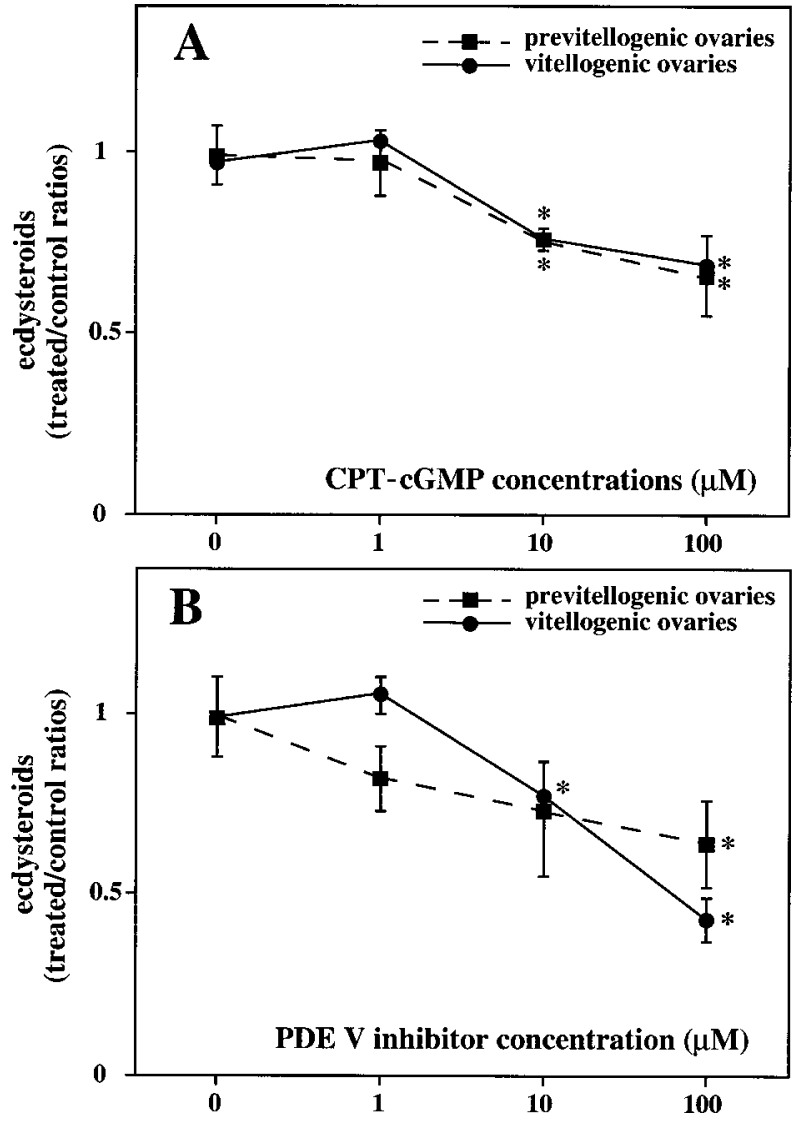

Figure 2 Effect of (A) CTP-CGMP, a cGMP analog, and of (B) MDBAMQ, a selective PDE $\mathrm{V}$ inhibitor, on ecdysteroid biosynthesis (treated/control ratios) by previtellogenic (broken lines) and vitellogenic (solid lines) ovaries, after $3 \mathrm{~h}$ of incubation. Each point represents the mean \pm S.E.M. obtained from seven to fifteen pairs of ovaries: from each female fly, one ovary of the pair was incubated with a known (or null for blanks) concentration (in $\mu \mathrm{M}$ ) of respective pharmacological compounds, whereas the contralateral one was used as its corresponding untreated control. Asterisks above error bars indicate results significantly different from the theoretical value of 1 (Student's $t$-test, $P<0 \cdot 05$ ).

vidual ovaries (one per pair) at the time of extirpation (made at different times after a liver meal) and the steroidogenic capacity of corresponding contralateral ovaries, incubated for $3 \mathrm{~h}$ in vitro: results (Fig. 4) showed that a peak in ovarian cGMP concentrations at the end of the vitellogenic cycle was associated with a dramatic decrease in steroidogenic capacity in vitro.

Correlations between cGMP and cAMP concentrations were also examined at the beginning of the vitellogenic cycle, our previous studies having established that the stimulation of steroidogenesis is associated with a peak in cAMP concentrations during the first $3 \mathrm{~h}$ after a protein meal, then followed by a return to basal levels during the rest of the cycle (Manière et al. 2000). Results (Fig. 5) demonstrated that no drop of cGMP was associated with 


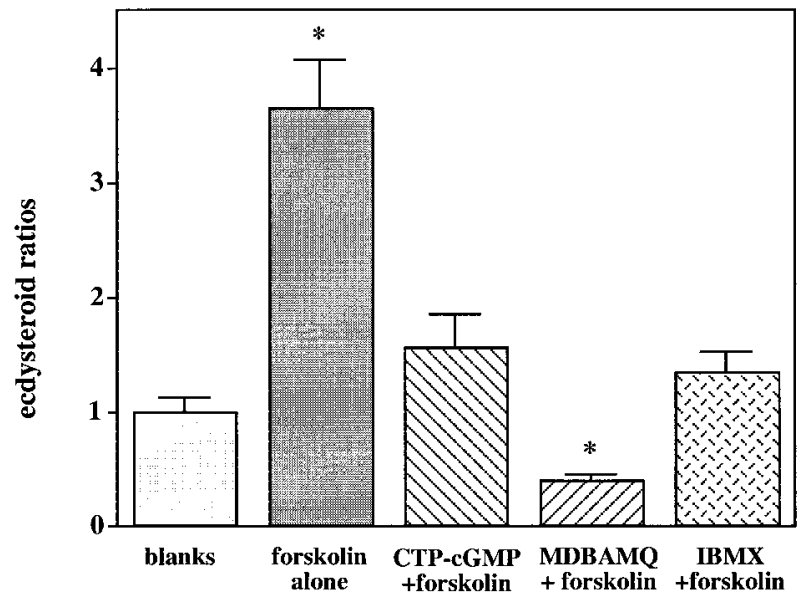

Figure 3 Effects of forskolin $\left(10^{-5} \mathrm{M}\right)$, either alone or in combination with CTP-cGMP $\left(10^{-4} \mathrm{M}\right)$, MDBAMQ $\left(10^{-4} \mathrm{M}\right)$ or IBMX $\left(10^{-3} \mathrm{M}\right)$, on ecdysteroid secretions by previtellogenic ovaries incubated for $3 \mathrm{~h}$ in Grace's insect culture medium. Each bar represents a mean \pm S.E.M. obtained from eight pairs of ovaries: from each female fly, pharmaceutical compound(s) was added to one ovary of the pair, whereas the contralateral one was used as its corresponding untreated control. Ecdysteroids were measured by immunoassay for each ovary. Results are expressed on a relative scale, as treated/control ratios (ordinate). A series of blanks was also incubated without any drug addition (blanks) to verify the regularity of ecdysteroid secretions between untreated ovaries extirpated from similar flies. Asterisks above error bars indicate results significantly different from the theoretical value of 1 (Student's $t$-test, $P<0 \cdot 05$ ). When expressed in absolute amounts, ecdysteroids secreted after a 3 -h incubation of untreated ovaries (blanks) represent $11 \cdot 4 \pm 1 \cdot 1 \mathrm{fmol}$ ecdysone equivalents per ovary $(n=16)$.

cAMP and ecdysteroid increases during the $3 \mathrm{~h}$ following a protein meal: unlike cAMP and ecdysteroids, cGMP values measured from samples taken 1 or $3 \mathrm{~h}$ after the protein meal did not significantly differ from those taken at time 0 (Student's $t$-test, $P>0 \cdot 05$ ).

\section{cGMP targets}

It is generally admitted (for reviews see Vaandrager \& de Jonge 1996, Lucas et al. 2000) that major targets for cGMP include cGMP-regulated PDE isoforms, cyclic nucleotidegated ion channels and/or protein kinase G (PKG). We have thus briefly examined these three possibilities. First, we have studied the possibility of a direct activation of a type II PDE (i.e. a cGMP-stimulated, cAMP-specific PDE), in order to investigate whether cGMP could exert its inhibitory effect by antagonizing cAMP signaling: for this purpose, experiments were performed with erythro9-(2-hydroxy-3-nonyl)adenine (EHNA), a potent inhibitor of type II PDEs (Mery et al. 1995, Podzuweit et al. 1995). Results showed that EHNA (tested from $10^{-6}$ to $10^{-4} \mathrm{M}$ ) was not able to increase cAMP and ecdysteroid biosyntheses in previtellogenic ovaries (for example, a dose

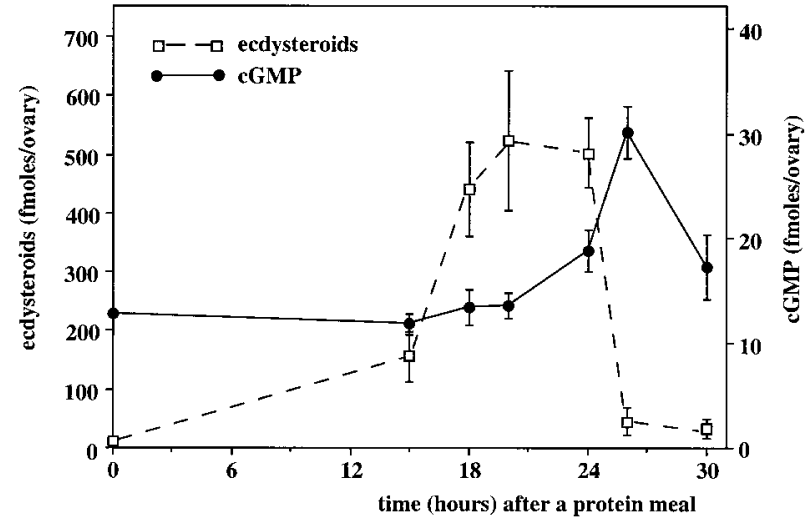

Figure 4 Temporal correlation between ovarian contents of cGMP, measured immediately after extirpation (expressed in fmol/ovary, right ordinate) and ecdysteroids secreted after an in vitro incubation of $3 \mathrm{~h}$ following extirpation (expressed in ecdysone equivalents per ovary, left ordinate). The abscissa represents the time of extirpation, expressed in hours after the beginning of a protein meal. Each point represents the mean \pm S.E.M. from four to eight individual measurements.

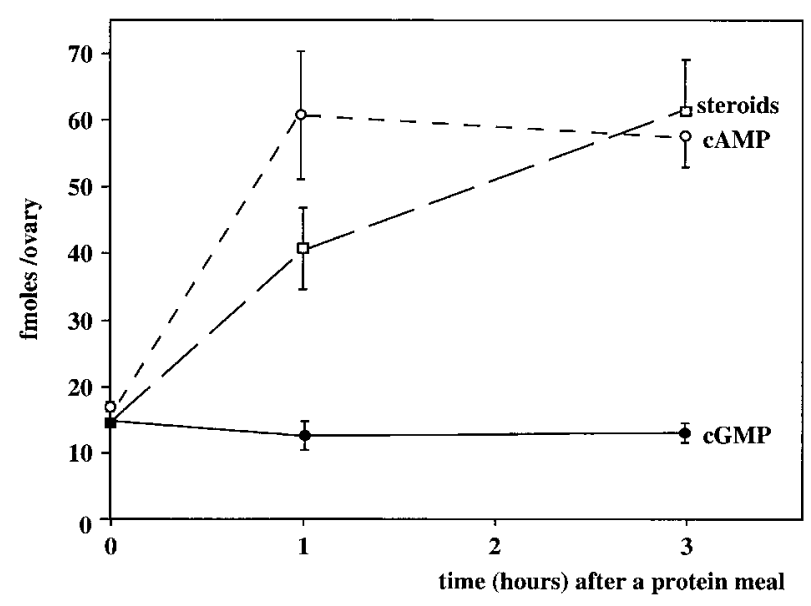

Figure 5 Evolution during the $3 \mathrm{~h}$ following a protein meal (time of ovarian extirpation on the abscissa) of the ovarian quantities (expressed in fmol/ovary, ordinate) of both cyclic nucleotides (measured immediately after extirpation) and of ecdysteroids secreted in the culture medium (measured after an in vitro incubation of $3 \mathrm{~h}$ following extirpation). Each point represents the mean \pm S.E.M. from five to eight individual ovaries.

of $10^{-4} \mathrm{M}$ EHNA tested on five to seven pairs of ovaries gave a cAMP treated/control ratio of $0.84 \pm 0.09 \quad(n=5)$ and an ecdysteroid treated/control ratio of $0.84 \pm 0.15$ $(n=7)$, results not significantly different from $1, P>0 \cdot 05)$. EHNA also failed to increase ecdysteroid biosynthesis after activation of previtellogenic ovaries by $10^{-5} \mathrm{M}$ forskolin, as well as failing to prevent the inhibition of steroidogenesis by MDBAMQ (data not shown). Altogether, these results did not corroborate the hypothesis of a cGMP effect on cAMP concentrations via the involvement of PDE II. The possibility of cGMP action on a type III PDE (i.e. a 
cGMP-inhibited PDE), though unlikely considering our other results, was nevertheless investigated using milrinone, a specific inhibitor of PDE III: this compound also failed to alter ecdysteroid biosynthesis (data not shown), thus suggesting an absence of control of cGMP on PDEs in blowfly ovary.

The possibility of an action of cGMP on cyclic nucleotide-gated calcium channels was then examined. Considering that, in blowfly ovary, contrary to what happens generally in other models, an influx of external calcium is able to decrease cAMP concentrations into steroidogenic cells by activating a calmodulin-sensitive PDE (Manière et al. 2002), an indirect action of cGMP on cAMP concentrations via the regulation of calcium influx might have been suspected. However, CTP-cGMP and MDBAMQ were found to keep their inhibiting properties on steroidogenesis in spite of the incubation of blowfly ovaries in calcium-free saline (a treatment previously found to eliminate extracellular calcium trapped within the ovary; see Manière et al. 2002): for example, CTPcGMP or MDBAMQ at $10^{-4} \mathrm{M}$ reduced ecdysteroid treated/control ratios of previtellogenic ovaries to respectively $0.65 \pm 0.08(n=7)$ or $0.32 \pm 0.09(n=8)$ in calciumfree saline. Such values were significantly different from 1 (Student's $t$-test, $P<0 \cdot 05$ ), but not significantly different from those observed in a calcium-containing medium (see Fig. 2 at $100 \mu \mathrm{M})$. Thus, these results showed that cGMP does not act on steroidogenesis through the activation of a cyclic nucleotide-gated calcium channel.

Lastly, experiments were carried out to investigate the involvement of PKG. By adding a specific PKG inhibitor, KT 5823, a significant increase of ecdysteroid biosynthesis was triggered in previtellogenic and, more particularly, in vitellogenic ovaries (Table 1). Together, these results thus showed that PKG is the main, if not unique, target of cGMP for the regulation of ovarian steroidogenesis.

\section{Potential cGMP activators}

Experiments were then carried out to examine some possible factors which could activate the cGMP-signaling pathway. First, the ability of brain factors to stimulate cGMP biosynthesis was tested on ovaries incubated in vitro. For this purpose, crude brain extracts were prepared, either from previtellogenic or vitellogenic females, and added to in vitro cultures of ovaries also obtained from both types of females. Table 2 shows that, whatever the stage combination between ovaries and brain extracts, no significant effect was observed on ovarian cGMP biosynthesis (note that, in these experiments, the effects on ovarian ecdysteroid secretion were not examined, as it has been previously demonstrated that similar brain extracts strongly activate cAMP and ecdysteroid biosyntheses, see Manière et al. 2000). Our experiments thus showed that fly brain probably does not elaborate ecdysteroidostatic neurohormones acting on ovaries via cGMP, and consequently
Table 1 Effect of KT 5823, a PKG inhibitor, on ecdysteroid biosynthesis in previtellogenic (sugar-fed) and vitellogenic (liver-fed, $24 \mathrm{~h}$ after meal) flies. Each concentration of inhibitor was tested on 5 to $17(n)$ flies. Ovaries of each pair were incubated separately in Grace's medium: one ovary was considered as a control, the other was given a known concentration of inhibitor (treated ovary). Using immunoassays, measurements of ecdysteroids were made after $3 \mathrm{~h}$ of incubation. Results are expressed as means \pm S.E.M. of individual treated/control ratios

\begin{tabular}{|c|c|c|c|}
\hline & $\begin{array}{l}\text { KT } 5823 \\
\text { concentrations }\end{array}$ & $n$ & $\begin{array}{l}\text { Ecdysteroids } \\
\text { (treated/contro } \\
\text { ratios) }\end{array}$ \\
\hline \multicolumn{4}{|l|}{ Ovary stage } \\
\hline Previtellogenic & $\begin{array}{l}10^{-7} \mathrm{M} \\
10^{-6} \mathrm{M} \\
10^{-5} \mathrm{M}\end{array}$ & $\begin{array}{r}5 \\
8 \\
17\end{array}$ & $\begin{array}{l}0 \cdot 88 \pm 0 \cdot 31 \\
1 \cdot 10 \pm 0 \cdot 29 \\
1 \cdot 61 \pm 0 \cdot 29^{*}\end{array}$ \\
\hline Vitellogenic & $\begin{array}{l}10^{-7} \mathrm{M} \\
10^{-6} \mathrm{M} \\
10^{-5} \mathrm{M}\end{array}$ & $\begin{array}{r}5 \\
6 \\
10\end{array}$ & $\begin{array}{l}0.91 \pm 0.31 \\
1 \cdot 66 \pm 0.25^{*} \\
2 \cdot 32 \pm 0.46^{*}\end{array}$ \\
\hline
\end{tabular}

*Results significantly different from the ideal value of 1 (Student's $t$-test, $P<0 \cdot 05)$.

suggest that this second messenger is rather activated by abdominal or intra-ovarian factor(s), probably involving a paracrine/autocrine mechanism.

As our previous observations had shown that calcium influx into ovarian cells (provoked by A23187 ionophore treatment) was a powerful inhibitor of steroidogenesis in our model (Manière et al. 2002), the following experiment was carried out to examine the effect of calcium on ovarian cGMP content. Vitellogenic ovaries were incubated with known concentrations of A23187 and their cGMP contents were compared with untreated corresponding controls. The results showed that A23187, at concentrations which drastically inhibited ecdysteroid secretion (from $10^{-5} \mathrm{M}$,

Table 2 Effect of crude brain extracts on cGMP biosynthesis by fly ovaries in vitro. Brain and ovaries were obtained from either previtellogenic (sugar-fed) or vitellogenic (liver-fed, $24 \mathrm{~h}$ after meal) flies. Crude brain extracts were added at a dose of three brain equivalents per $50 \mu \mathrm{l}$ culture medium (as previously described in Manière et al. 2000). Each line of results was obtained from the ovaries of four individual flies. From each fly, one ovary was incubated in the presence of brain extract (treated), the other in its absence (control). Incubation time: $1 \mathrm{~h}$. Individual measurements of cGMP are expressed as treated/control ratios (means \pm S.E.M.)

cGMP biosynthesis (treated/control ratios)

\begin{tabular}{lll}
\cline { 3 - 3 } Brain extract stage & Ovary stage & \\
Previtellogenic & Previtellogenic & $1 \cdot 21 \pm 0 \cdot 34$ \\
Previtellogenic & Vitellogenic & $0 \cdot 90 \pm 0 \cdot 23$ \\
Vitellogenic & Previtellogenic & $1 \cdot 14 \pm 0 \cdot 34$ \\
Vitellogenic & Vitellogenic & $0 \cdot 98 \pm 0 \cdot 10$
\end{tabular}

Values did not significantly differ from the ideal value of 1 (Student's $t$-test, $P>0 \cdot 05)$. 


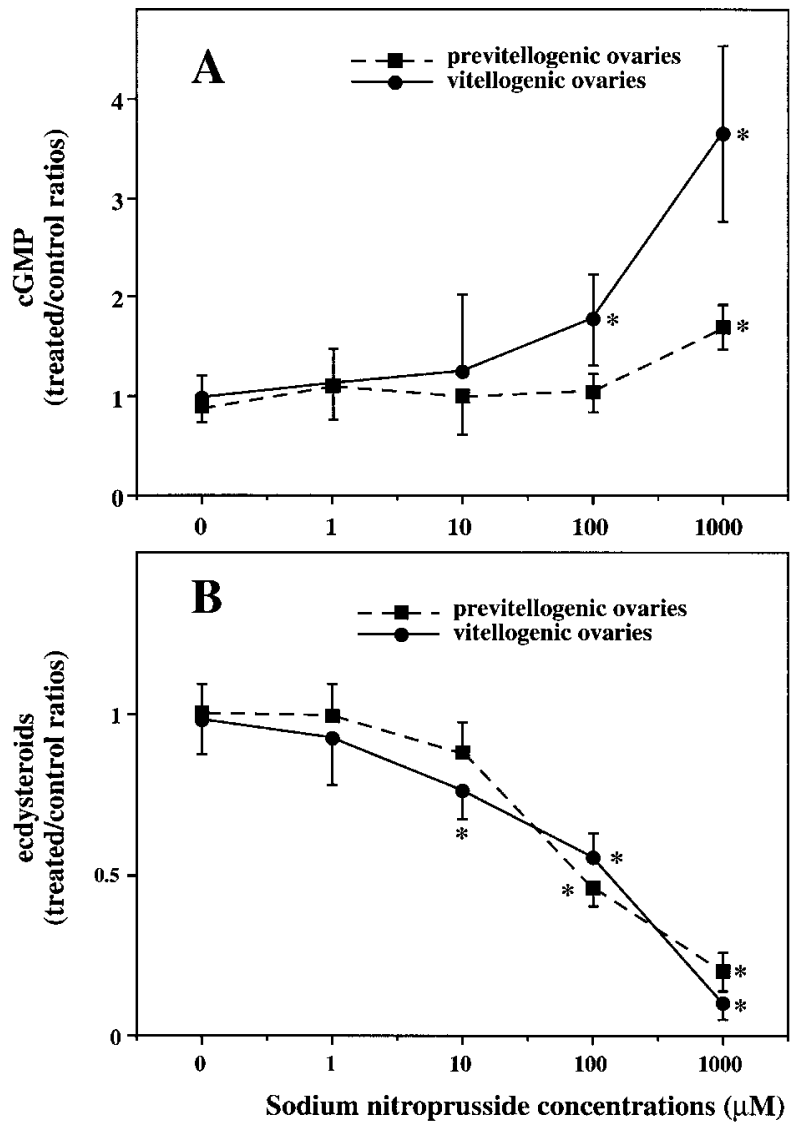

Figure 6 In vitro effects of sodium nitroprusside (concentrations in $\mu \mathrm{M}$ on the abscissa) on (A) cGMP ovarian levels and (B) secretion of ecdysteroids, obtained from incubations of previtellogenic (broken lines) and vitellogenic (solid lines) ovaries. Incubation times: $1 \mathrm{~h}$ for cGMP, $3 \mathrm{~h}$ for ecdysteroids. Each point represents the mean \pm S.E.M. of treated/control ratios obtained from five to thirteen pairs of ovaries. Asterisks indicate values significantly different from the theoretical value of 1 (Student's $t$-test, $P<0 \cdot 05)$.

see Manière et al. 2002), did not significantly increase the ovarian levels of cGMP: for example, treatment with $10^{-5} \mathrm{M} \mathrm{A} 23187$ corresponded to a cGMP concentration ratio of $1 \cdot 27 \pm 0 \cdot 30(n=8)$ and treatment with $10^{-4} \mathrm{M}$ to $1 \cdot 44 \pm 0 \cdot 44(n=7)$, ratios which were not significantly different from the theoretical value of 1 (Student's $t$-test, $P>0.05)$. These data thus suggest that cGMP is not involved in the regulation of steroidogenesis by calcium.

Lastly, the possibility of control of cGMP concentrations by $\mathrm{NO}$ was examined with experiments using sodium nitroprusside, a potent source of NO. When added to the incubation medium of previtellogenic or of vitellogenic ovaries (Fig. 6), this compound was found to significantly enhance cGMP biosynthesis (from $10^{-4} \mathrm{M}$ ) and also to inhibit ecdysteroidogenesis (from $10^{-5} \mathrm{M}$ ). Thus, a possible steroidostatic effect of $\mathrm{NO}$ via the involvement of cGMP can be hypothesized in blowfly ovary.

\section{Discussion}

The IBMX paradox and the regulation of steroidogenesis by cyclic nucleotides

The presence of cGMP in blowfly ovary and its capacity to inhibit ovarian steroidogenesis was revealed by the careful examination of the effects of IBMX. As this PDE inhibitor was able to increase the ovarian concentrations of both cyclic nucleotides in our model, the absence of its effect on steroidogenesis may not be explained by the presence of IBMX-insensitive PDEs, but suggests that the steroidogenic effect of cAMP is prevented by cGMP. Indeed, our present experiments, showing that a cGMP analog is able to inhibit ovarian steroidogenesis and that a specific PDE $\mathrm{V}$ inhibitor has a powerful steroidostatic effect, allow an explanation of the IBMX paradox by the opposite effects of both cyclic nucleotides. This interpretation is confirmed by the observation of an inhibitory capacity of IBMX (as well as of other cGMP-mimicking compounds) when associated with a cAMP-mimicking substance such as forskolin.

Thus, cGMP- and cAMP-signaling pathways constitute, in blowfly ovarian follicles, two opposite mechanisms of the control of steroidogenesis. The existence of such a regulation by cyclic nucleotides is described here for the first time in an invertebrate model. Indeed, in insect molting (prothoracic) glands for example, the cAMP pathway is generally known to have a stimulatory role (for review see Gilbert et al. 1996), though sometimes possible inhibitory effects (Henrich 1995, Hua \& Koolman 1995). The presence of cGMP has not been detected so far in prothoracic glands (Vedeckis et al. 1976). Moreover, cGMP analogs seem to have weak or null effects (Smith et al. 1984, Hua \& Koolman 1995) and IBMX induces the same responses as cAMP analogs. Another series of examples is found in crustacean molting glands where both cyclic nucleotides seem to have inhibitory effects (Sedlmeier \& Fenrich 1993, Spaziani et al. 1999): indeed, they probably co-operate in inhibiting steroidogenesis (Saïdi et al. 1994) and act through a single protein kinase (Baghdassarian et al. 1996), so that the pharmacological effects of IBMX on steroidogenesis in crustacean molting glands seem to be always inhibitory. From the above comparisons (as well as from others, detailed below), the regulation of steroidogenesis appears to be more complex in blowfly ovaries than in insect or crustacean molting glands.

\section{Possible activators of cGMP}

Several peptidic factors have been reported to inhibit ecdysteroid biosynthesis in the molting glands of arthropods. Beside MIH in crustaceans (for review see Keller 1992), several prothoracicostatic factors (generally named ecdysiostatins) have also been characterized in 
insects (Hua et al. 1994, 1999). However, no peptide has been isolated so far for its capacity to inhibit steroidogenesis in the gonads of arthropods, though partial purifications have suggested that such factors exist in flies and are probably elaborated in the abdomen (Adams \& Li 1998). Our own experiments, which failed to show a regulation of ovarian cGMP by brain extracts, suggest that a cerebral control is unlikely and support the hypothesis of paracrine or autocrine regulations.

In mammals, NO has been described as an autocrine/ paracrine regulator of ovarian steroidogenesis (Van Voorhis et al. 1994). NO signaling has been observed in several insect tissues (for review see Davies 2000), but, to the best of our knowledge, not yet in gonads. The above results have shown that an NO donor can be a powerful activator of cGMP biosynthesis in Phormia ovaries, indicating the presence of an NO-sensitive soluble guanylate cyclase. Moreover, an NO donor is also an efficient inhibitor of ecdysteroidogenesis, which is in agreement with a regulation of ovarian steroidogenesis by NO-cGMP signaling. However, these results are not sufficient to definitively conclude that NO could physiologically regulate ecdysteroid biosynthesis via cGMP in blowfly ovaries. Indeed, it has been frequently observed in vertebrates that NO (and NO donors) can have inhibitory effects on steroidogenesis by acting directly on P-450 biosynthetic enzymes and not necessarily via the cGMP pathway (Van Voorhis et al. 1994). Our preliminary experiments in blowfly ovaries with an inhibitor of NO-sensitive soluble guanylate cyclases did not prevent alterations of ecdysteroid biosynthesis by NO (G Manière, unpublished observations). Moreover, it remains to be demonstrated in our model that NO is really produced near or inside ovarian follicules. Other preliminary experiments using the NADPH-diaphorase staining method (G Manière, unpublished observations) have indicated the presence of $\mathrm{NO}$-synthase in the blowfly ovary, mainly located in a few egg chambers which prematurately aborted vitellogenesis, thus suggesting a possible role in the regulation of egg chamber degeneration (which participates in the control of the number of mature oocytes and may influence steroidogenesis). However, a more precise investigation of the sites and the timing of NO production, as well as of the location of soluble guanylate cyclase, is needed to better analyse the exact role of $\mathrm{NO}$ during ovarian ecdysteroidogenesis in Phormia.

Calcium is also frequently known to be a regulator of NO-synthase, as well as of guanylate cyclase (for review see Stuehr 1999, Lucas et al. 2000). In a previous study (Manière et al. 2002), we have postulated the existence of an ecdysteroidostatic factor acting on ovarian steroidogenesis via calcium signaling. It could be hypothesized that the same factor may also be responsible for the involvement of cGMP. However, our present experiments demonstrated, on one hand, that cGMP signaling remains active in a calcium-free medium (which makes improbable a regu- lation of steroidogenesis by cGMP via the control of calcium influx) and, on the other hand, they also indicated that calcium influx has no effect on cGMP levels (which makes a control of cGMP concentrations by calcium unlikely). Thus, our results are not in agreement with the existence of a single negative regulation involving both calcium and cGMP, but suggest the presence of two separate inhibitory pathways. This hypothesis is reinforced by the fact that calcium inhibition is known, in our model, to alter the cAMP pathway (because of the involvement of a calciumsensitive type I PDE, Manière et al. 2002), whereas our present results indicate that cGMP failed to regulate cAMP concentrations. Nevertheless, other investigations also remain necessary to better understand the relationships between calcium and cGMP in blowfly ovary.

\section{cGMP during the reproductive cycle}

Our present experiments clearly indicated that the cGMPsignaling system (possibly linked with $\mathrm{NO}$ ) is always present and is able to react at any time during the reproductive cycle, in previtellogenic as well as in vitellogenic ovaries. Similarly, our previous observations on calcium inhibition have also demonstrated its efficiency in previtellogenic and in vitellogenic flies (Manière et al. 2002). Interestingly, this dual inhibitory control is opposed to (at least) a dual stimulatory regulation, involving not only a cAMP-dependent pathway but also a cAMPindependent mechanism (Manière et al. 2000), which seems to involve insulin-like signaling (G Manière \& J-P Delbecque, unpublished obervations). So, a minimum of four different types of regulatory pathways, at least two inhibitory (maybe more if NO constitutes a separate pathway) and two stimulatory, control ovarian steroidogenesis during the blowfly reproductive cycle.

In previtellogenic flies, these different regulations seem to maintain a complex equilibrium which keeps steroidogenesis at a basal but significant rate, from which it can be rapidly down- or up-regulated. Experiments with IBMX have shown that when two opposite mechanisms were put simultaneously into action with a similar intensity, they did not break this equilibrium. There is no doubt that, beyond the artefactual effects of IBMX, this capacity is probably very important in physiological conditions, as it may delay vitellogenesis under unfavorable conditions.

However, this equilibrium has to disappear for the stimulation of steroidogenesis and the consequential initiation of vitellogenesis. Indeed, at this step, a complete desynchronization was particularly observed for the two cyclic nucleotide pathways (nothing being presently known for the other mechanisms): our results indeed showed that cGMP concentrations remained constant during the stimulation of steroidogenesis, whereas cAMP levels increased significantly. This seems to be different from what is observed in crustacean molting glands, in which the stimulation of steroidogenesis needs the removal 
of the inhibitory control by cyclic nucleotides (Spaziani et al. 1999), but appears to be in agreement with observations in the mammal reproductive cycle (Ling et al. 1980).

At the end of vitellogenesis, our experiments showed that cGMP reached its maximal levels, in correlation with the decline of steroidogenesis in vitro. A correlation was also observed in vivo between cGMP ovarian levels and hemolymph titers of ecdysteroids (authors' unpublished data). However, in this case, the decline in circulating ecdysteroids appeared to occur a few hours later than in vitro, approximately at the time of the cGMP peak. Such a delay is not surprising, because, as observed in the mosquito or in several other species (for review see Hagedorn 1989), including our model (authors' unpublished observations), stimulated ovaries generally secrete a precursor such as ecdysone into the hemolymph, then this precursor is transformed by other organs into the active hormone, 20-hydroxyecdysone, which is the main circulating and active hormone and which is then transformed into inactive (but immunodetected) metabolites. Thus, the delay between the peak of steroidogenesis in vitro and in vivo can be easily interpreted by the time necessary for these biochemical transformations and by the respective half-lifes of these different ecdysteroids in the hemolymph.

The involvement of both cyclic nucleotides in the termination of normal vitellogenesis has been previously observed in the follicles of a lepidopteran species, Hyalophora cecropia and, more particularly, it was demonstrated that they regulate vitellogenin uptake (Wang \& Telfer 1996). In Phormia, it seems unlikely that cAMP could play such a role due to its very low levels during this period (Manière et al. 2000), but our experiments strongly suggest that cGMP plays a major role in the termination of vitellogenesis by inhibiting ovarian steroidogenesis and, consequently, stopping the stimulation of vitellogenin biosynthesis by the fat body. Nevertheless, this interpretation does not exclude the possibility of a complementary role of cGMP on the control of vitellogenin uptake.

The very low levels of cAMP (Manière et al. 2000) at the time of the cGMP peak could also suggest that cGMP is able to down-regulate cAMP concentrations. However, such a possibility was not confirmed by our experiments, which failed to demonstrate that cGMP could stimulate cAMP degradation by specific PDEs. These arguments, however, must be considered with caution, because they are partly based upon the absence of effects of a pharmaceutical compound, EHNA, which is known to be active in mammals, but which has not been extensively studied in insects. Nevertheless, although cAMP and cGMP pathways appear to be antagonistic, no evidence has been obtained during this study that they really interact or alter each other's concentrations. However, as demonstrated previously for cAMP (Manière et al. 2000) and in this study for cGMP, both cyclic nucleotides seem to act essentially through their respective protein kinases: it is thus probable that the antagonistic actions of the two signaling systems only intervene downstream from these kinases and not through a reciprocal regulation of their own levels.

Our data thus show that, contrary to the situation in previtellogenic flies, the two cyclic nucleotide-signaling pathways do not intervene simultaneously and symmetrically during vitellogenesis, but separately and alternatively: this difference in phase is undoubtedly essential for the regulation and completion of a normal vitellogenic cycle. Inversely, it can be hypothesized that a precocious activation of cGMP (or of $\mathrm{NO}$ or $\mathrm{Ca}^{2+}$ as well) before the completion of the reproductive cycle would probably result in an incomplete vitellogenesis. In this context, it would be interesting to verify the alterations of cAMP and cGMP pathways in blowflies with naturally or experimentally abortive vitellogeneses.

Finally, the involvement of cGMP in the regulation of steroidogenesis during the whole reproductive cycle appears unequivocal in blowfly ovary, in opposition to the complex situation observed in vertebrates (see Introduction). The involvement of NO in this regulation appears probable but remains to be fully demonstrated. Although it is premature to suggest that cGMP (and possibly NO) could have conserved their roles in the control of ovarian cycles during evolution, such a hypothesis deserves to be examined by further studies in other invertebrate models.

\section{Acknowledgements}

We are indebted to Dr M De Reggi (Marseille, France) and Dr T Kingan (Riverside, CA, USA) for the gift of antibodies or reagents for immunoassays. We also thank $\mathrm{Cl}$ Renouleaud for her valuable technical help.

\section{References}

Adams TS \& Li QJ 1998 Ecdysteroidostatin from the house fly, Musca domestica. Archives of Insect Biochemistry and Physiology 38 166-176.

Baghdassarian D, de Besse N, Saidi B, Somme G \& Lachaise F 1996 Neuropeptide-induced inhibition of steroidogenesis in crab molting glands: involvement of cGMP-dependent protein kinase. General and Comparative Endocrinology 104 41-51.

Bredt DS \& Snyder SH 1994 Nitric oxide: a physiologic messenger molecule. Annual Review of Biochemistry 63 175-195.

Cooke BA 1999 Signal transduction involving cyclic AMP-dependent and cyclic AMP-independent mechanisms in the control of steroidogenesis. Molecular and Cellular Endocrinology 151 25-35.

Davies S 2000 Nitric oxide signalling in insects. Insect Biochemistry and Molecular Biology $301123-1138$.

Delbecque JP, Weidner K \& Hoffmann KH 1990 Alternative sites for ecdysteroid production in insects. Invertebrate Reproduction and Development 18 29-42.

Del Punta K, Charreau EH \& Pignataro OP 1996 Nitric oxide inhibits Leydig cell steroidogenesis. Endocrinology 137 5337-5343.

Drewett JG \& Garbers DL 1994 The family of guanylyl cyclase receptors and their ligands. Endocrine Reviews 15 135-162. 
Gilbert LI, Rybczynski R \& Tobe SS 1996 Endocrine cascade in insect metamorphosis. In Metamorphosis, pp 59-107. Eds LI Gilbert, JR Tata \& BG Atkinson. San Diego, CA: Academic Press Inc.

Grasselli F, Ponderato N, Basini G \& Tamanini C 2001 Nitric oxide synthase expression and nitric oxide/cyclic GMP pathway in swine granulosa cells. Domestic Animal Endocrinology 20 241-252.

Hagedorn HH 1985 The role of ecdysteroids in reproduction. In Comprehensive Insect Physiology, Biochemistry and Pharmacology, vol. 8, pp 205-262. Eds GA Kerkut \& LI Gilbert. Oxford, UK: Pergamon Press.

Hagedorn H 1989 Physiological roles of hemolymph ecdysteroids in the adult insect. In Ecdysone. From Chemistry to Mode of Action, pp 279-289. Ed. J Koolman. Stuttgart: Georg Thieme Verlag.

Hagedorn H, O'Connor JD, Fuchs MS, Sage B, Schlager DA \& Bohm MK 1975 The ovary as a source of $\alpha$-ecdysone in an adult mosquito. PNAS 72 3255-3259.

Henrich VC 1995 Comparison of ecdysteroid production in Drosophila and Manduca: pharmacology and cross-species neural reactivity. Archives of Insect Biochemistry and Physiology 30 239-254.

Hua YJ \& Koolman J 1995 An ecdysiostatin from flies. Regulatory Peptides 57 263-271.

Hua YJ, Bylemans D, De Loof A \& Koolman J 1994 Inhibition of ecdysone biosynthesis in flies by a hexapeptide isolated from vitellogenic ovaries. Molecular and Cellular Endocrinology 104 R1-R4.

Hua YJ, Tanaka Y, Nakamura K, Sakakibara M, Nagata S \& Kataoka H 1999 Identification of a prothoracicostatic peptide in the larval brain of the silkworm, Bombyx mori. Journal of Biological Chemistry 274 31169-31173.

Ishimaru RS, Leung K, Hong L \& LaPolt PS 2001 Inhibitory effects of nitric oxide on estrogen production and cAMP levels in rat granulosa cell cultures. Journal of Endocrinology 168 249-255.

Keller R 1992 Crustacean neuropeptides: structures, functions and comparative aspects. Experientia 48 439-448.

Khurana ML \& Pandey KN 1993 Receptor-mediated stimulatory effect of atrial natriuretic factor, brain natriuretic peptide, and C-type natriuretic peptide on testosterone production in purified mouse Leydig cells: activation of cholesterol side-chain cleavage enzyme. Endocrinology 133 2141-2149.

Kingan TG 1989 A competitive enzyme-linked immunosorbent assay: applications in the assay of peptides, steroids and cyclic nucleotides. Analytical Biochemistry 183 283-289.

LaPolt PS \& Hong H-S 1995 Inhibitory effects of superoxide dismutase and cyclic guanosine $3^{\prime}, 5^{\prime}$-monophosphate on estrogen production in cultured rat granulosa cells. Endocrinology 136 5533-5539.

Ling WY, Williams MT \& Marsh JM 1980 Correlation of cyclic AMP and cyclic GMP accumulation and steroidogenesis during stimulation of bovine luteal cells with luteinizing hormone. Journal of Endocrinology 86 45-52.

Loeb MJ, Woods CW, Brandt EP \& Borkovec AB 1982 Larval testes of the tobacco budworm. A new source of insect ecdysteroids. Science 218 896-898.

Lucas KA, Pitari GM, Kazerounian S, Ruiz-Stewart I, Park J, Schulz S, Chepenik KP \& Waldman SA 2000 Guanylyl cyclases and signaling by cyclic GMP. Pharmacological Reviews 52 375-414.

Manière G, Vanhems E \& Delbecque JP 2000 Cyclic AMP-dependent and -independent stimulations of ovarian steroidogenesis by brain factors in the blowfly, Phormia regina. Molecular and Cellular Endocrinology 168 31-40.

Manière G, Vanhems E, Gautron F \& Delbecque JP 2002 Calcium inhibits ovarian steroidogenesis in the blowfly Phormia regina. Journal of Endocrinology 173 533-544.

Masuda M, Kubota T, Karnada S \& Aso T 1997 Nitric oxide inhibits steroidogenesis in cultured porcine granulosa cells. Molecular Human Reproduction 3 285-292.
Mery PF, Pavoine C, Pecker F \& Fischmeister R 1995 Erythro-9-(2-hydroxy-3-nonyl)adenine inhibits cyclic GMP-stimulated phosphodiesterase in isolated cardiac myocytes. Molecular Pharmacology 48 121-130.

Mukhopadhyay AK, Schumacher M \& Leidenberger FA 1986 Steroidogenic effect of atrial natriuretic factor in isolated mouse Leydig cells is mediated by cyclic GMP. Biochemical Journal 239 463-467.

Pascual N, Bellés X, Delbecque JP, Hua YJ \& Koolman J 1995 Quantification of ecdysteroids by immunoassay: comparison of enzyme immunoassay and radioimmunoassay. Zeitschrift für Naturforschung 50c 862-867.

Podzuweit T, Nennstiel P \& Muller A 1995 Isozyme selective inhibition of cGMP-stimulated cyclic nucleotide phosphodiesterases by erythro-9-(2-hydroxy-3-nonyl) adenine. Cellular Signalling 7 733-738

Saïdi B, De Bessé N, Webster SG, Sedlmeier D \& Lachaise F 1994 Involvement of cAMP and cGMP in the mode of action of molt inhibiting hormone (MIH) a neuropeptide which inhibits steroidogenesis in a crab. Molecular and Cellular Endocrinology 102 $53-61$.

Schumacher H, Muller D \& Mukhopadhyay AK 1992 Stimulation of testosterone production by atrial natriuretic peptide in isolated mouse Leydig cells results from a promiscuous activation of cyclic AMP-dependent protein kinase by cyclic GMP. Molecular and Cellular Endocrinology 90 47-52.

Sedlmeier D \& Fenrich R 1993 Regulation of ecdysteroid biosynthesis in crayfish Y-organs: I. Role of cyclic nucleotides. Journal of Experimental Zoology 265 448-453.

Smith WA, Gilbert LI \& Bollenbacher WE 1984 The role of cyclic AMP in the regulation of ecdysone synthesis. Molecular and Cellular Endocrinology 37 285-294.

Spaziani E, Mattson MP, Wang WNL \& McDougall HE 1999 Signaling pathways for ecdysteroid hormone synthesis in crustacean Y-organs. American Zoologist 39 496-512.

Stuehr DJ 1999 Mammalian nitric oxide synthases. Biochimica et Biophysica Acta - Bioenergetics 1411 217-230.

Takase Y, Saeki T, Watanabe N, Adachi H, Souda S \& Saito I 1994 Cyclic GMP phosphodiesterase inhibitors. 2. Requirement of 6-substitution of quinazoline derivatives for potent and selective inhibitory activity. Journal of Medicinal Chemistry 37 2106-2111.

Vaandrager AB \& de Jonge HR 1996 Signalling by cGMP-dependent protein kinases. Molecular and Cellular Biochemistry 157 23-30.

Van Voorhis BJ, Dunn MS, Snyder GD \& Weiner CP 1994 Nitric oxide: an autocrine regulator of human granulosa luteal cell steroidogenesis. Endocrinology 135 1799-1806.

Vedeckis WV, Bollenbacher WE \& Gilbert LI 1976 Insect prothoracic glands: a role for cyclic AMP in the stimulation of $\alpha$-ecdysone secretion. Molecular and Cellular Endocrinology 5 81-88.

Wang Y \& Telfer WH 1996 Cyclic nucleotide-induced termination of vitellogenin uptake by Hyalophora cecropia follicles. Insect Biochemistry and Molecular Biology 26 85-94.

Welch C, Watson ME, Poth M, Hong T \& Francis GL 1995 Evidence to suggest nitric oxide is an interstitial regulator of Leydig cell steroidogenesis. Metabolism 44 234-238.

Received 2 December 2002

Accepted 10 December 2002 